\title{
Absolute Stereochemistry Determination of Tetrin B
}

\author{
Geonseek Rỵu, Byoung Wook Choi, ${ }^{\dagger}$ and Bong Ho Leet ${ }^{\dagger+}$ \\ Actranced Haterial Research Center for Better Emiromment. \\ Deparment of Chemical Techology, Hanhat National Linversity. 16-1 Dukmung-dong, \\ Iusming-gu. Dajon 305-719. Korea \\ Received duly \& 2002
}

\begin{abstract}
The absolute stereochemistry of tetrin $\mathrm{B}(\mathbf{1})$, an antifungal antibiotic isolated from a soil actinonycete was determined by applying Rychnorsky analysis, the modified Mosher method, and (D) exciton chirality to be $4 R$, $5 R, 7 S, 9 R, 115,12 R .13 S, 15 K, 24 S$, and $25 R$.
\end{abstract}

Key Words : Tetrin B. Absolute stereochenistry

A novel antifungal antibiotic tetrin B (1) was isolated from a soil actinomycete in 1963 and its structure was finally established after a number of erroneous proposals. ${ }^{1-3}$ Although tetrin $B$ was ascertained to belong to the 26-membered tetraene macrolide. its stereochemistry has remained undefined. Spectroscopic and synthetic inrestigations of the stereochemistry of tetrin-type macrocy cles have been carried out mainly for pimaricin. ${ }^{3-5}$ A controlled degradation protocol designed for stereostructural studies was developed to lead a $\mathrm{C} 3-\mathrm{Cl} 6$ segment. a possible target for its synthetic work by Fraser-Reid's group. ${ }^{6}$ Duplantier and Masamune reported stereochemical studies based on the reagent controlled asymmetric synthesis of the diastereomers, which also defined the stereochemistry of pimarolide, thus representing the first synthetic access to this type of macrocycles. Recently. Beau's group determined the complete configuration of pimaricin on the basis of coupling constants $(J)$ derived from muclear magnetic resonance and qualitative NOEs." Nevertheless. such efforts have not given the absolute configuration of tetrin B. More recently, we isolated tetrin-related compounds from Streptomyces sp. GK 924 . $^{9}$ In this paper we describe the complete stereochemistry of its major metabolite tetrin $\mathrm{B}$.

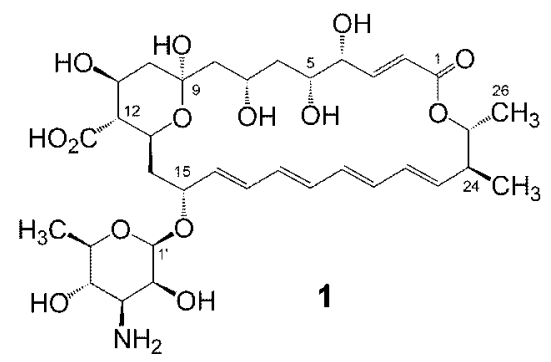

A method to determine the absolute configuration of $\mathbf{1}$ was developed (Scheme 1). First. compound 1 was treated with $1.0 \mathrm{~N} \mathrm{NaOH}$ to afford sodium dicarboxy late and pentaenal 4. The dicarboxylate was immediately methylated with diazomethane to give a methyl diester 3 . which was

"Corresponding Nuthor: Phone: +82-42-821-1542, Fax: +82-42822-1562, c-mail: lbhol liathanbal.ac.kr subsequently converted to two acetonides (5. 6). The gross structure of 5 was readily identified by the mass and NMR spectral data. The chemical shifts of the acetonide ketal $(\delta$ 99.3) and two methyl carbons (30.1. 19.9) ${ }^{\text {li) }}$ and the coupling constants between the methine protons ( $\mathrm{H} 5$ and $\mathrm{H} 7$ ) in the six membered dioxane ring of $\mathbf{5}$ suggested that the acetonide of a sw-1,3-diol had been produced (Figure 1). The oxymethine proton (H5) signal at $3.87 \mathrm{ppm}$ showed $2.9 \mathrm{~Hz}$ and $10.1 \mathrm{~Hz}$ couplings to the $\mathrm{H} \mathrm{s}_{\mathrm{c}}$ and $\mathrm{H} \mathrm{6}_{\mathrm{ax}}$ signal at 1.12 ppm and $1.36 \mathrm{ppm}$, respectively. and another oxymethine proton $(\mathrm{H} 7)$ at 3.74 ppm was coupled to $\mathrm{H} 6_{\mathrm{ec}}$ and $\mathrm{H} 6_{\mathrm{ax}}$ to 2.0 $\mathrm{Hz}$ and $11.8 \mathrm{~Hz}$ values. respectively. The magnitude of these values. which reflects the chair conformation of the acetonide ring. indicates that both $\mathrm{H} 5$ and $\mathrm{H} 7$ are anti to $\mathrm{H} 6_{\mathrm{ax}}$. As expected in this conformation. $\mathrm{H} 5$ and $\mathrm{H} 7$ signals exhibited strong NOEs to the acetonide methyl ( $\mathrm{k} n$ to $\mathrm{H} 5$ and $\mathrm{H} 7$ ) signal at $0.81 \mathrm{ppm}$. whereas the $\mathrm{H}_{\mathrm{ax}}$ signal displayed no NOE correlation with other protons in the dioxane ring. We concluded that the relative configurations at $\mathrm{C} 5$ and $\mathrm{C} 7$ in 5 are $5 R^{*}$ and $75^{*}$. The relative stereochemistry of $\mathrm{C}+$ and $\mathrm{C} 5$ in 1 was established by NMR data for acetonide 6 (Fig. 1$)$; a coupling constant $\left(J_{\mathrm{H}-\mathrm{H} .5}=8.1 \mathrm{~Hz}\right.$ ) and the NOE correlations between $\mathrm{H} 5$ and $\mathrm{H} 3$ and between $\mathrm{H} 6 \mathrm{a}$ and $\mathrm{H}+\mathrm{in} 6$ indicated a syn relationship between two hydroxyl groups at $\mathrm{C} 4$ and $\mathrm{C}^{5}$. This was consistent with an empirical rule for assigning the stereochemistry of 1.2acetonides by chemical shifts of methyl groups: both methyls of the isopropylidene unit in 6 resonated at 1.38 $1.39 \mathrm{ppm} .{ }^{11}$ thereby, resulting in $+R * 5 R^{*}$-configuration.

The absolute stereochemistry determination of two acetonides (5. 6) was achieved by the modified Mosher's method. ${ }^{\text {I2 }}$ The $(R)$ - and $(S)$-MTPA [2-methoxy-2-(trifluoromethyl)-2phenyl acetyl] derivatives ( $7 \mathbf{a}$ and $7 \mathbf{b}$. and $8 \mathbf{a}$ and $8 \mathbf{b}$ ) were prepared. and $\Delta \delta\left(\delta_{\mathrm{s}}-\delta_{\mathrm{R}}\right)$ values for all the assignable protons were determined with $400 \mathrm{MHz}$ NMR (Fig. 2). The absolute configurations of $\mathrm{C}+$ in 5 and $\mathrm{Cll}$ in 6 were indicated as $R$ and $S$, respectively. Furthemore. $C D$ analysis of di-p-bromobenzoate $\mathbf{1 1}$ afforded more evident absolute configurations of $\mathrm{C}+$ and $\mathrm{C} 5$ in 6 . A series of hydrogenation $\left(\mathrm{H}_{2} / \mathrm{Pd}\right)$, acetylation ( $\left.\mathrm{Ac}_{2} \mathrm{O} / \mathrm{pyr}\right)$. acidic hydrolysis $(80 \%$ aqueous $\mathrm{AcOH})$, and $p$-bromobenzoy lation $\left(p-\mathrm{BrC}_{6} \mathrm{H}_{4} \mathrm{COCV}\right.$ 


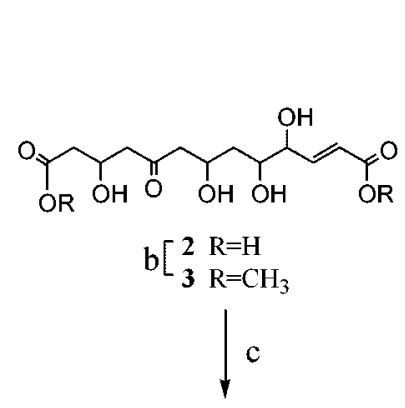<smiles>COC(=O)/C=C/C(O)C1CC(CC(=O)CC(O)CC(=O)OC)OC(C)(C)O1</smiles><smiles>COC(=O)CC(O)CC(=O)CC(O)CC1OC2C(C(=O)OC)CC(C)(O1)C2(C)C</smiles>
6 d<smiles>CCOC(C=CC(=O)OC)C1CC(CC(=O)CC(O)CC(=O)OC)OC(C)(C)O1</smiles>

Scheme 1. a: 1 .0 $\mathrm{N} \mathrm{NaOH}$ b: $\mathrm{CH}_{2} \mathrm{~N}_{2} / \mathrm{F}, \mathrm{t}_{2} \mathrm{O}$, c: 2,2-DMT. CSA,

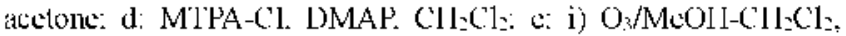
NaBIi, ii) LiAlli: l: i) LiOll, MeOll-11_O: ii) 2,2-LMP, (SA. acetone

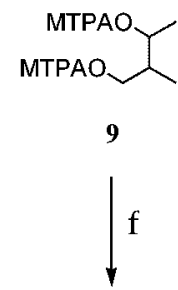<smiles>CC1COC(C)(C)OC1C</smiles>

10

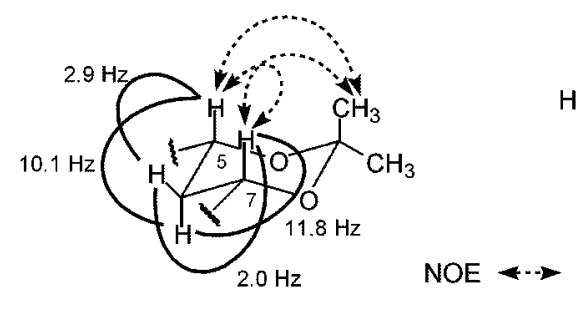

5

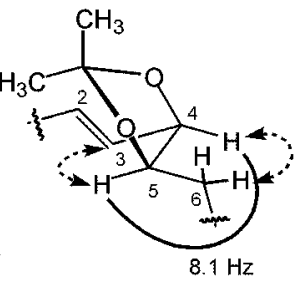

6

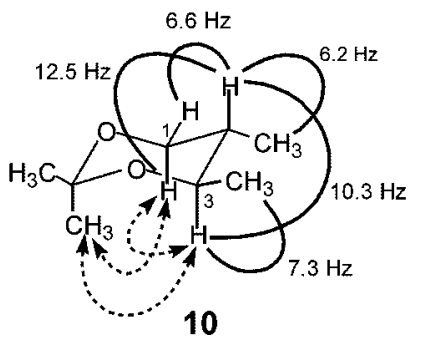

Figure 1, Coupling constants and NOE correlations providing widence for relative conliguration and confomedion of the acetonide in 5, 6, and 10 .

pyr) of 6 produced a saturated di- $p$-bromobenzoate derivative 11 (Scheme 2). The CD spectrum of 11 showed a clear negative exciton split [first Cotton at $255 \mathrm{lum}(\Delta \varepsilon=-6.5)$ : second Cotton at $239 \mathrm{~nm}(\Delta \varepsilon=+7.1) \mathrm{J}$. and thus the $+R$ and $5 R$ configurations were confirmed unambignously. On the other hand. the relative stereochemistry of the carboxyl group-substituted tetrahydropyran ring of $\mathbf{1}$ was assigned by NOESY experiments in the preceding paper. ${ }^{9}$ The absolute stereochemistry of the tetrahydropyran ring in 1 was determined as $9 R, 11 S .12 R$, and $13 \mathrm{~S}$

Determination of the absolute configurations at $\mathrm{C} 2+$ and $\mathrm{C} 25$ in 1 was achiered by NMR experiments for a degraded fragment of 4 . Sequential ozonolysis $\left(\mathrm{O}_{3} / \mathrm{MeOH}-\mathrm{CH}_{2} \mathrm{Cl}_{2}\right.$. $\left.\mathrm{NaBH}_{1}\right)$ of 4 and reduction $\left(\mathrm{LiAlH}_{1}\right)$ efficiently produced a<smiles>[R6]O[C@@H](C)[C@H](C)[C@@H](C)O[R6]</smiles>

9a $\mathrm{R}=(+)-(R)-\mathrm{MTPA}$

9b $\mathrm{R}=(-)-(S)-\mathrm{MTPA}$

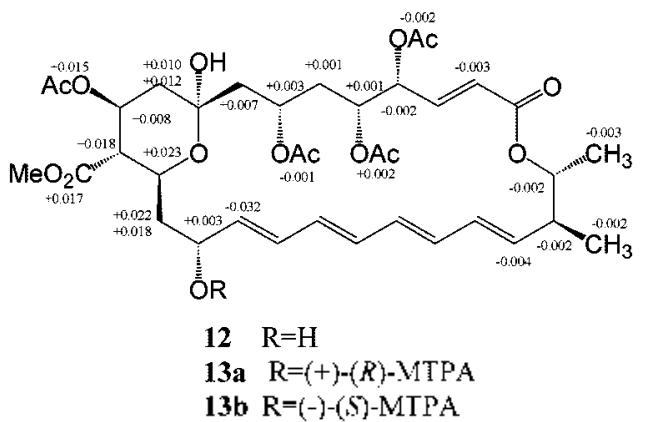

Figure 2. $\Delta \delta=\Delta_{S} \Delta_{R}$ values in ppm obtained at 400 IL for the MTPA esters 7a. 7b. 8a. 8b. 9a. 9b. 13a, and 13b. 


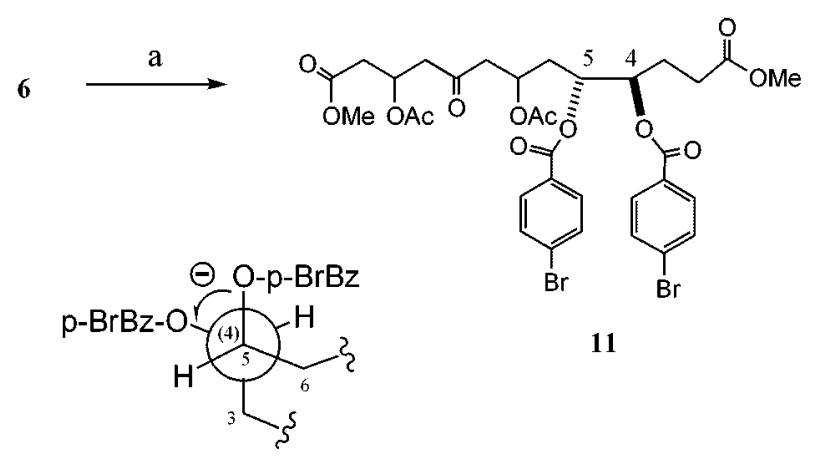

Scheme 2. a: i) $\mathrm{H}_{3}, \mathrm{Pd} / \mathrm{C}, \mathrm{MeOH}$ : ii) $\Lambda \mathrm{c}_{2} \mathrm{O}$, py, r.t. iii) $80 \% \wedge \mathrm{cOH}$. $\left.60)^{\circ} \mathrm{C}: \mathrm{iv}\right) \mathrm{p}-\mathrm{BrC} \mathrm{CH}, \mathrm{COCl} \mathrm{py} \cdot 40^{\circ} \mathrm{C}$.

1.3-diol. which was treated with MTPA-Cl to give MTPA diesters (9a and 9b). The modified Mosher analysis established the $R$-configuration at $\mathrm{C} 3$ (Fig. 2). To determine the configuration of $\mathrm{C} 2$ in 9 , the combined MTPA diesters (9a and 9b) were treated with $\mathrm{LiOH}$ to afford the 1.3-diol. which was finally converted to acetonide $\mathbf{1 0}$. The coupling constant and NOE data of $\mathbf{1 0}$ provided the chair conformation and configuration of the acetonide ring (Fig. 1). The relative configurations at $C 2$ and $C 3$ in 10 were determined as $2 S^{*}$ and $3 R^{*}$ At this stage, the stereochemistry of $\mathrm{Cl}_{5}$ in 1 remained uncertain. A series of metlyylation $\left(\mathrm{CH}_{2} \mathrm{~N}_{-} / \mathrm{MeOH}\right)$. acetylation $\left(\mathrm{Ac}_{2} \mathrm{O} / \mathrm{py}\right)$, and acidic lydrolysis $(5 \% \mathrm{HCl} / \mathrm{MeOH})$ of 1 gave a deglycosylated tetracetyl derivative $\mathbf{1 2}$. Using the same steps, the $(R)$ and $(S)$-MTPA esters (13a and $\mathbf{1 3 b})$ were produced. and the $\Delta \delta\left(\delta_{\mathrm{s}}-\delta_{\mathrm{k}}\right)$ values indicated that the absolute configuration at $\mathrm{Cl} 15$ is $R$ (Fig. 2). Therefore. the absolute stereochemistry of 1 was determined as $+R, 5 R, 7 S, 9 R .11 S, 12 R, 13 S, 15 R$. $24 S$ and $25 R$.

The aminosugar mycosamine. as shown in the previous paper. $^{9}$ was established as 1)-series in comparison with the literature data. ${ }^{+}$

\section{Experimental Section}

Moisture and air sensitive reactions were performed in a flame-dried glassware equipped with rubber septa under positive nitrogen pressure. $\mathrm{Et}_{2} \mathrm{O}$ and $\mathrm{THF}$ were distilled from sodium benzophenone ketyl. $\mathrm{CH}_{2} \mathrm{Cl}_{2}$ and pyridine were also distilled from $\mathrm{CaH}_{2}$ before use. Kieselgel $60(0.063-0.2 \mathrm{~mm})$ was used for column chromatography and Merck Kieselgel $60 \mathrm{~F}_{25+}$ for TLC. HPLC was carried out on a Waters 510 apparatus equipped with a reversed-phase column (Cosmosil ODS, $5 \mu \mathrm{m} .10 \times 250 \mathrm{~mm}$ ). FAB mass spectra were measured on a JEOL JMX-SX 102 mass spectrometer. CD spectrum was measured on a JASCO J-20 Automatic Recording Spectropolarimeter. 'H-NMR and ${ }^{17} \mathrm{C}-\mathrm{NMR}$ spectra were recorded on a Brucker ARX- 400 spectrometer and chemical shifts are given in ppm relative to the solvent peaks $\left[\mathrm{CDCl}_{7}\right.$ $\left(\delta_{\mathrm{H}}=7.26\right.$ and $\left.\delta_{c}=77.1\right): \mathrm{CD}_{3} \mathrm{OD}\left(\delta_{\mathrm{H}}=3.30\right.$ and $\left.\left.\delta_{r}=49.0\right)\right]$ as internal standards.

Organisms and fermentation. Streptomyces sp. GK92+4 from a soil sample collected in Taejon. Koreal. was cultured in the seed medium consisting of glucose $2 \%$, starch $1 \%$, soybean flour $2.5 \%$, yeast extract $0.4 \%$. $\mathrm{NaCl} 0.2 \%$. $\mathrm{K}_{2} \mathrm{HPO}_{4} 0.005 \%$, and beef extract $0.1 \%$ (adjusted to $\mathrm{pH} 7.3$ before sterilization). The seed culture was carried out on a rotary shaker $(250 \mathrm{rpm})$ at $28^{\circ} \mathrm{C}$ for 24 hours in $500-\mathrm{mL}$ Erlenmeyer flasks containing $100 \mathrm{~mL}$ of the seed medium. Then, the seed culture $(100 \mathrm{~mL})$ was inoculated to a 50 -L jar fermenter containing $10 \mathrm{~L}$ of the production medium (antifoam $0.08 \%$ ). Fernentation was carried out at $27^{\circ} \mathrm{C}$ for 4 days with aeration $(10 \mathrm{~L} / \mathrm{min})$ under constant agitation $(250 \mathrm{rmm})$.

Extraction and isolation. The culture broth $(80 \mathrm{~L})$ was centrifuged to separate the mycelial cake. The mycelial cake was stirred ovenught in $70 \%$ aqueous acetone and filtered. The filtrate was concentrated in vacio to remove the organic solvent. resulting in an aqueous solution. The combined filtrates were passed through a Diaion HP-20 column. and washed with $\mathrm{H}_{2} \mathrm{O}$ followed by $\mathrm{MeOH}$. The $\mathrm{MeOH}$ eluate was partitioned between $\mathrm{CH}_{2} \mathrm{Cl}_{2}$ and $60 \%$ aqueous $\mathrm{MeOH}$. and then the $60 \%$ aqueous $\mathrm{MeOH}$ was re-partitioned between $n-\mathrm{BuOH}$ and $\mathrm{H}_{2} \mathrm{O}$. The $n-\mathrm{BuOH}$ fraction having antifungal activity was fractionated by ODS flash chromatography with aqueous $\mathrm{MeOH}$. The $70 \%$ aqueous $\mathrm{MeOH}$ fraction was further purified on Sephadex LH-20 with $\mathrm{MeOH}$ followed by reversed-phase HPLC with $63 \%$ aqueous $\mathrm{MeOH}$ to yield tetrin $\mathrm{B}(1.152 \mathrm{mg})$ as a major metabolite together with other tetrin-related compounds.

Methyl dicarboxylate 3 and pentaenal 4 . Tetrin $B(150$ $\mathrm{mg}$ ) in $5 \mathrm{~mL}$ of $1.0 \mathrm{~N}$ sodium hydroxide was stirred at room temperature overnight and extracted with ether several times. The water phase was neutralized with $\mathrm{I} \mathrm{N} \mathrm{HCl}$ and worked up with ethyl acetate to give a diacid residue (2). This residue was dried in a vacuum oven overnight and then immediately esterified with diazomethane. The reaction mixture was purified through a small silica gel column to afford a methyl dicarbosylate (3.63 mg). 3: FABMS (pos) $m z 3+9(\mathrm{M}+\mathrm{H}):{ }^{1} \mathrm{H} \mathrm{NMR}\left(\mathrm{CDCl}_{3}, 400 \mathrm{MHz}\right) \delta 5.77(1 \mathrm{H}$. dd. $J=15.4 .5 .6 \mathrm{~Hz}, \mathrm{H}-3), 5.51$ (lH. d. $J=15.4 \mathrm{~Hz} . \mathrm{H}-2)$. $4.0 \mathrm{I}$ ( $1 \mathrm{H}$, dd. $J=7.2,5.6 \mathrm{~Hz}, \mathrm{H}-4) .3 .66(1 \mathrm{H}, \mathrm{m} . \mathrm{H}-1 \mathrm{l}) .3 .47$ (IH. m. H-5). $3.4 \mathrm{l}(\mathrm{lH}, \mathrm{m} . \mathrm{H}-7), 3.2 \mathrm{l}\left(3 \mathrm{H}, \mathrm{s} .13-\mathrm{OCH}_{3}\right)$, $3.12\left(3 \mathrm{H}, \mathrm{s}, 1-\mathrm{OCH}_{3}\right) .2 .38(\mathrm{lH} . \mathrm{dd} . J=12.6 .3 .8 \mathrm{~Hz}, \mathrm{H}-8)$. $2.72(\mathrm{lH} . \mathrm{dd} . J=12.6 .8 .6 \mathrm{~Hz} . \mathrm{H}-8) .2 .40(1 \mathrm{H} . \mathrm{dd} . J=12.3$. $8.2 \mathrm{~Hz}, \mathrm{H}-10$ ). 2.18 ( $\mathrm{lH} . \mathrm{dd} . J=13.2 .6 .9 \mathrm{~Hz} \mathrm{H}-12$ ). 2.08 (lH. dd. $J=13.2,4.2 \mathrm{~Hz} . \mathrm{H}-12), 1.91(\mathrm{lH} . \mathrm{dd} . J=12.3 .4 .1$ Hz. H-10). $1 .+7$ (lH. m. H-6). 1.26 (lH. m. H-6).

The ether phase was worked up to give a yellow residue (56 mg). which was purified by preparative TLC $[20 \%$ AcOEt/hexane]. Pentaenal $(4,42 \mathrm{mg})$ was recrystallized from cyclohexane as a major product. 4 : FABMS (pos) $m: z$ $255(\mathrm{M}+\mathrm{Nal}))^{-}:{ }^{\mathrm{H}} \mathrm{NMR}\left(\mathrm{CDCl}_{\text {.. }} 400 \mathrm{MHz}\right) \delta 9.42(1 \mathrm{H}, \mathrm{d} . J$ $=8.7 \mathrm{~Hz} . \mathrm{H}-1) .7 .17(\mathrm{lH} . \mathrm{dd} . J=15.2 .7 .8 \mathrm{~Hz} \cdot \mathrm{H}-3) \cdot 6.2-6.9$ (7H. complex). $6.1+(1 \mathrm{H} . \mathrm{dd} J=15.2,8.7 \mathrm{~Hz} . \mathrm{H}-2), 5.67$ (IH. dd. $J=15.8 .8 .4 \mathrm{~Hz}, \mathrm{H}-\mathrm{Il}$ ). 3.80 (lH. m. H-13). 2.12 $(\mathrm{H}, \mathrm{m} . \mathrm{H}-12) .1 .28(3 \mathrm{H}, \mathrm{d} . J=6.1 \mathrm{~Hz}, \mathrm{H}-\mathrm{l}+), 1.19$ (3H. d. $J$ $=6.0 \mathrm{~Hz} .12-\mathrm{CH}_{3}$ ).

Acetonides 5 and 6. A solution of the methyl dicarboxylate $3(58 \mathrm{mg})$ dissolved in a $4: 1$ mixture $(3 \mathrm{~mL})$ of acetone 
and 2,2-dimethoxypropane (DMP) was treated with camphor sulfonic acid (CSA, $5 \mathrm{mg}$ ). The reaction was stimed under $\mathrm{N}_{2}$ at room temperature for $l \mathrm{~h}$. The reaction was then quenched with $\mathrm{Et}_{3} \mathrm{~N}(0.5 \mathrm{~mL})$ and concentrated under a stream of $\mathrm{N}_{z}$. Silica gel chromatography ( $15 \%$ AcOEt/hexane) gave an acetonide mixtıre (46 mg). which was purified by reversed-phase HPLC (94\% aqueous $\mathrm{MeOH}$ ) to give 5 (18 $\mathrm{mg}$ ) and 6 (22 $\mathrm{mg}$ ). respectively. 5: FABMS (pos) $m z 389$ $(\mathrm{M}+\mathrm{H}){ }^{1}:{ }^{1} \mathrm{H} \mathrm{NMR}\left(\mathrm{CDCl}_{3}, 400 \mathrm{MHz}\right) \delta 6.7+(1 \mathrm{H}, \mathrm{dd}, J=$ 15.6. $7 .+\mathrm{Hz}, \mathrm{H}-3) .5 .68(\mathrm{lH}, \mathrm{d} . J=15.6 \mathrm{~Hz}, \mathrm{H}-2) .4 .10(1 \mathrm{H}$. dd. $J=7.4 .4 .6 \mathrm{~Hz}, \mathrm{H}-4$ ). 3.87 (lH. ddd. $J=10.1 .4 .6 .2 .9$ Hz. H-5), $3.7+$ (lH. m. H-7), 3.63 (lH. m. H-ll). $3.18(3 \mathrm{H}$, s. $\left.13-\mathrm{OCH}_{3}\right), 3.14\left(3 \mathrm{H}\right.$. s, $\left.1-\mathrm{OCH}_{3}\right), 2.38(\mathrm{lH} . \mathrm{dd} . J=13.8$, $8.6 \mathrm{~Hz} . \mathrm{H}-10), 2.22$ (lH, dd, $f=12.8 .6 .8 \mathrm{~Hz}, \mathrm{H}-12) .2 .12$ (1H. dd. $J=13.8 .2 .8 \mathrm{~Hz} . \mathrm{H}-10) .2 .01$ (1H. dd. $J=14.2 .3 .4$ Hz. H-8), 1.92 (lH. dd. $J=12.8 .4 .2 \mathrm{~Hz} . \mathrm{H}-12), 1.78(1 \mathrm{H}$. dd. $J=1+.2,8.2 \mathrm{~Hz}, \mathrm{H}-8) .1 .4 \mathrm{l}\left(3 \mathrm{H}, \mathrm{s}\right.$. acetonide $\left.\mathrm{CH}_{3}\right), 1.36$ (1H. ddd. $\left.J=14.6 .11 .8 .10 .1 \mathrm{~Hz}, \mathrm{H}-6_{i k}\right), 1.12$ (1H, ddd, $J=$ 14.6. 2.9. $\left.2.0 \mathrm{~Hz} . \mathrm{H}-6_{\text {cul }}\right) .0 .81$ (3H. s, acetonide $\left.\mathrm{CH}_{3}\right) ;{ }^{1.3} \mathrm{C}$ NMR NMR ( $\left.\mathrm{CDCl}_{3 .}+00 \mathrm{MHz}\right) \delta 206.3$ (s, C-9). 168.4 (s, C-1). 164.2 (s. C-13). 144.3 (d, C-3). 123.6 (d, C-2). 99.3 (s, ketal C), 68.4 (d. C-4). 66.9 (d. C-11), 62.6 (d, C-5). 61.9 (d. C-7). 52.6 (q. $13-\mathrm{OCH}_{3}$ ). 51.8 (q. $1-\mathrm{OCH}_{3}$ ). 41.2 (t. C-10), 42.3 (t. C-8). 39.3 (t, C-6). 37.4 (t, C-12), 30.1 (q. acetonide $\mathrm{CH}_{3}$ ). 19.9 (q. acetonide $\mathrm{CH}_{3}$ ). 6: FABMS (pos) $m z+11$ $(\mathrm{M}+\mathrm{Na})^{\prime} .389(\mathrm{M}+\mathrm{H})^{\prime}:{ }^{\prime} \mathrm{H} \mathrm{NMR}\left(\mathrm{CDCl}_{3 .}+00 \mathrm{MHz}\right) \delta 6.81$ (lH. dd. $J=15.4 .8 .1 \mathrm{~Hz} . \mathrm{H}-3), 5.52(\mathrm{lH}, \mathrm{d}, J=15.4 \mathrm{~Hz} . \mathrm{H}-$ 2). 4.18 (lH. t. $J=8.1 \mathrm{~Hz} . \mathrm{H}-4$ ). 3.92 (lH. m. H-5). 3.48 (lH. m. H-1l). 3.34 (lH. m. H-7). 3.15 (3H. s, l-OCH $\mathrm{OCH}_{3}$, $3.12\left(3 \mathrm{H}, \mathrm{s} .13-\mathrm{OCH}_{3}\right), 2.13(\mathrm{lH}, \mathrm{dd} . J=13.8 .6 .6 \mathrm{~Hz}, \mathrm{H}-8)$. $2.0 \mathrm{l}$ ( $1 \mathrm{H}$, dd $J=13.2,6.8 \mathrm{~Hz}, \mathrm{H}-12) .1 .92$ ( $1 \mathrm{H}, \mathrm{dd}, J=12.6$, $6.2 \mathrm{~Hz} . \mathrm{H}-10), 1.83$ (1H. dd. $J=13.2 .4 .1 \mathrm{~Hz}, \mathrm{H}-12) .1 .74$ (lH. dd $J=13.8 .4 .2 \mathrm{~Hz}, \mathrm{H}-8), 1.70$ (lH. m. H-lo), 1.62 (lH. ddd. $J=13.2,7.9 .6 .+\mathrm{Hz} . \mathrm{H}-6$ ). 1.39 (3H. s. acetoride $\mathrm{CH}_{3}$ ). 1.38 (3H. s, acetonide $\mathrm{CH}_{3}$ ). 1.30 (lH. ddd. $J=13.2$. 4.9. $2.2 \mathrm{~Hz}, \mathrm{H}-6)$

MTPA esters 7 a and $7 \mathrm{~b}$. A small amount of acetonide 5 (3 $\mathrm{mg}$ ) dissolved in $2 \mathrm{~mL}$ of $\mathrm{CH}_{2} \mathrm{Cl}_{2}$ was treated with DMAP (5 mg) and (R)-MTPA-Cl (0.1 mL). After stirring overnight. the reaction was quenched with saturated $\mathrm{NaHCO}_{\S}$ solution and extracted with $\mathrm{CH}_{2} \mathrm{Cl}_{2}$. The organic layer was washed with $\mathrm{H}_{2} \mathrm{O}$ and brine. The residue was dried $\left(\mathrm{Na}_{2} \mathrm{SO}_{4}\right)$ and concentrated under reduced pressure. Silica gel chromatography ( $10 \%$ AcOEt/hexane) gave MTPA ester mixtures, of which the major component was isolated by reveresd-phase HPLC (95\% aqueous $\mathrm{MeOH})$ to afford an $(R)$-MTPA ester $7 \mathrm{a}(2.8 \mathrm{mg}) .7 \mathrm{a}:$ FABMS (pos) $m z 605(\mathrm{M}+\mathrm{H}){ }^{1}:{ }^{\prime} \mathrm{H}$ NMR (CDCl $\left.{ }_{\text {in }} 400 \mathrm{MHz}\right) \delta 7.405(5 \mathrm{H}$, complex, MTPA-phenyl). 6.801 (lH. dd. $J=15.6 .7 .1 \mathrm{~Hz} . \mathrm{H}-3$ ) .5 .722 (lH. d. $J=15.6$ Hz. H-2) $4.83+$ (1H. dd. $J=7.1 .4 .6 \mathrm{~Hz} . \mathrm{H}-4), 3.957(\mathrm{lH}$. ddd. $J=10.8 .+6.2 .9 \mathrm{~Hz} \cdot \mathrm{H}-5) .3 .895(1 \mathrm{H} . \mathrm{m} \cdot \mathrm{H}-7) .3 .7 \mathrm{I} 2$ (lH. m. H-11) 3.529 (3H. s. MTPA-OCH $\left.13-\mathrm{OCH}_{3}\right), 3.065\left(3 \mathrm{H} . \mathrm{s} .1-\mathrm{OCH}_{3}\right) .2 .382(1 \mathrm{H} . \mathrm{dd}, J=13.8$. $8.6 \mathrm{~Hz}, \mathrm{H}-10$ ). $2.26 \mathrm{l}$ (lH. dd. $J=13.4 .4 .2 \mathrm{~Hz}, \mathrm{H}-\mathrm{I} 2$ ) $.2 .10 \mathrm{l}$ (1H. dd. $J=13.8 .2 .8 \mathrm{~Hz} . \mathrm{H}-10), 2.043$ (1H. dd. $J=1+.2 .3 .4$ Hz. H-8). 1.934 (lH. dd. $J=13.4 .6 .8 \mathrm{~Hz} . \mathrm{H}-12$ ). $1.82 \mathrm{l}$ ( $1 \mathrm{H}$. dd. $J=1+.2 .8 .1 \mathrm{~Hz}, \mathrm{H}-8), 1.392(1 \mathrm{H}$. ddd. $J=1+.6,10.8$.
$8.2 \mathrm{~Hz}, \mathrm{H}-6$ ). 1.185 (lH. ddd, $f=14.6 .2 .9 .2 .0 \mathrm{~Hz}, \mathrm{H}-6$ ). 1.036 (3H. s. acetonide $\mathrm{CH}_{3}$ ), 0.837 (3H. s. acetonide $\mathrm{CH}_{3}$ ).

On the other hand. the (S)-MTPA ester was prepared from acetolide 5 ( $3 \mathrm{mg}$ ) by the same procedure used to prepare the ( $R$ )-MTPA ester 7 a described above. The reaction mixture was purified by a series of silica gel cluromatography ( $10 \%$ AcOEt/hexane) and ODS HPLC $(95 \% \mathrm{MeOH})$ to yield an (S)-MTPA ester $7 \mathbf{b}(2.3 \mathrm{mg}) .7 \mathbf{b}$ : FABMS (pos) $m z 605(\mathrm{M}+\mathrm{H}):{ }^{1} \mathrm{H} \mathrm{NMR}\left(\mathrm{CDCl}_{3}, 400 \mathrm{MHz}\right) \delta 7.408(5 \mathrm{H}$, complex. MTPA-phenyl), $6.833(\mathrm{lH}$, dd, $f=15.6 .7 .1 \mathrm{~Hz}$. $\mathrm{H}-3) .5 .746$ ( $\mathrm{lH}, \mathrm{d}, J=15.6 \mathrm{~Hz}, \mathrm{H}-2), 4.838(\mathrm{lH} . \mathrm{dd}, J=7.1$, $4.6 \mathrm{~Hz}, \mathrm{H}-4$ ). 3.935 (1H. ddd, $f=10.8 .4 .6 \mathrm{~Hz} .2 .9, \mathrm{H}-5$ ). 3.879 (lH. m. H-7). 3.705 (lH. m. H-ll). 3.529 (3H. s, MTPA-OCH 3 ). 3.147 (3H, s. 13-OCH $) .3 .087$ (3H, s. 1$\left.\mathrm{OCH}_{3}\right), 2.371(\mathrm{lH} . \mathrm{dd}, j=13.8,8.6 \mathrm{~Hz} . \mathrm{H}-10) .2 .25+(1 \mathrm{H}$, dd, $J=13.4 .4 .2 \mathrm{~Hz}, \mathrm{H}-12), 2.092$ (1H, dd. $f=13.8 .2 .8 \mathrm{~Hz}$. $\mathrm{H}-10), 2.025$ ( $\mathrm{H}$, dd. $J=14.2 .3 .4 \mathrm{~Hz} . \mathrm{H}-8), 1.928$ (1H. dd. $J=13.4,6.8 \mathrm{~Hz} . \mathrm{H}-12), 1.809$ (1H. dd, $J=14.2,8.1 \mathrm{~Hz}, \mathrm{H}-$ 8). $1.369(1 \mathrm{H} . \mathrm{ddd}, J=1+.6,10.8 .8 .2 \mathrm{~Hz} . \mathrm{H}-6), 1.165(1 \mathrm{H}$, ddd. $J=14.6,2.9 .2 .0 \mathrm{~Hz} . \mathrm{H}-6) .1 .012(3 \mathrm{H}, \mathrm{s}$, acetonide $\left.\mathrm{CH}_{3}\right), 0.820\left(3 \mathrm{H}, \mathrm{s}\right.$. acetonide $\left.\mathrm{CH}_{3}\right)$

MTPA esters 8a and 8b. Preparation of $(R)-\mathrm{MTPA}$ ester $8 \mathrm{a}(2.2 \mathrm{mg})$ from acetolude 6 (3 $\mathrm{mg})$ as a starting material was achieved in the same procedure as 7a. 8a: FABMS (pos) $m z 627(\mathrm{M}+\mathrm{Na}) .605(\mathrm{M}+\mathrm{H})^{\prime}$ : 'H NMR $\left(\mathrm{CDCl}_{3} .400\right.$ $\mathrm{MHz}) \delta 7.38+(5 \mathrm{H}$, complex. MTPA-phenyl). 6.822 (1H. dd. $j=15.4 .8 .1 \mathrm{~Hz}, \mathrm{H}-3) .5 .541$ (1H. d. $j=15.4 \mathrm{~Hz} . \mathrm{H}-2)$, $4.623(\mathrm{lH}, \mathrm{m}, \mathrm{H}-\mathrm{ll}), 4.177$ (lH. t. $J=8.1 \mathrm{~Hz}, \mathrm{H}-4) .3 .942$ (lH. t. $J=8.1 \mathrm{~Hz}, \mathrm{H}-5), 3.548\left(3 \mathrm{H} . \mathrm{s} . \mathrm{MTPA}-\mathrm{OCH}_{3}\right) .3 .204$ (lH. m. H-7). $3.164\left(3 \mathrm{H}\right.$, s. $\left.13-\mathrm{OCH}_{3}\right), 3.082$ (3H. s, 1$\left.\mathrm{OCH}_{3}\right), 2.115(\mathrm{lH} . \mathrm{dd} . J=13.8,6.7 \mathrm{~Hz} . \mathrm{H}-8) \cdot 2.104(\mathrm{lH} . \mathrm{dd}$. $J=13.2,6.9 \mathrm{~Hz} . \mathrm{H}-12), 1.996(\mathrm{lH} . \mathrm{dd}, J=12.6,6.3 \mathrm{~Hz} . \mathrm{H}-$ 10), 1.908 (lH. dd, $J=13.2,4.2 \mathrm{~Hz} . \mathrm{H}-12), 1.841$ (1H. m. $\mathrm{H}-10), 1.806(\mathrm{lH} . \mathrm{dd} . J=13.8,4.2 \mathrm{~Hz} . \mathrm{H}-8) .1 .714(1 \mathrm{H}$. ddd. $J=13.2,8.0 .6 .+\mathrm{Hz} . \mathrm{H}-6) .1 .396(3 \mathrm{H}, \mathrm{s}$, acetonide $\left.\mathrm{CH}_{3}\right), 1.301(\mathrm{H}, \mathrm{dd}, J=13.2 .5 .0 \mathrm{~Hz}, \mathrm{H}-6) .1 .383(3 \mathrm{H}, \mathrm{s}$. acetoluide $\left.\mathrm{CH}_{3}\right)$.

Preparation of $(R)$-MTPA ester $8 \mathbf{b}(1.8 \mathrm{mg})$ from acetonide 6 ( $3 \mathrm{mg}$ ) as a starting material was also achieved in the same procedure as 7b. 8b: FABMS (pos) $m z 627(\mathrm{M}+\mathrm{Na})$ : ${ }^{\prime} \mathrm{H}$ NMR $\left(\mathrm{CDCl}_{3,} 400 \mathrm{MHz}\right) \delta 7.38+(5 \mathrm{H}$. complex. MTPAphenv ), $6.81+$ (lH. dd. $J=15.4 .8 .1 \mathrm{~Hz}, \mathrm{H}-3$ ). 5.534 (lH. d. $j=15.4 \mathrm{~Hz}, \mathrm{H}-2)+.625(\mathrm{lH} . \mathrm{m} . \mathrm{H}-\mathrm{ll}),+.167(\mathrm{lH}, \mathrm{t}, J=8.1$ Hz. H-1). 3.926 (lH, t. $J=8.1 \mathrm{~Hz}, \mathrm{H}-5$ ). 3.548 (3H. s. MTPA-OCH $\left.{ }_{3}\right) .3 .384(1 \mathrm{H}, \mathrm{m} . \mathrm{H}-7) .3 .192\left(3 \mathrm{H}, \mathrm{s}, 13-\mathrm{OCH}_{3}\right)$. 3.075 (3H. s. $1-\mathrm{OCH}_{3}$ ). $2.09 \mathrm{l}(\mathrm{IH} . \mathrm{dd} . J=13.8 .6 .7 \mathrm{~Hz} . \mathrm{H}-$ 8). $2.123(1 \mathrm{H}$. dd. $J=13.2 .6 .9 \mathrm{~Hz} . \mathrm{H}-12) .1 .97+(\mathrm{lH} . \mathrm{dd} . J=$ 12.6. $6.3 \mathrm{~Hz} . \mathrm{H}-10) .1 .954(\mathrm{lH} . \mathrm{dd} . J=13.2 .4 .2 \mathrm{~Hz} . \mathrm{H}-12)$. $1.805(1 \mathrm{H}, \mathrm{m}, \mathrm{H}-10), 1.778$ (lH. dd, $J=13.8,+.2 \mathrm{~Hz}, \mathrm{H}-8$ ). 1.750 ( $1 \mathrm{H}$, ddd. $J=13.2,80.6 .4 \mathrm{~Hz}, \mathrm{H}-6), 1.376(3 \mathrm{H} . \mathrm{s}$, acetonide $\left.\mathrm{CH}_{3}\right), 1.347(\mathrm{lH} . \mathrm{dd} J=13.2 .4 .9 \mathrm{~Hz}, \mathrm{H}-6), 1.326$ (3H. s, acetonide $\mathrm{CH}_{3}$ )

MTPA diesters 9 a and 9 b. The yellow pentaenal $4(40$ mg) was dissolved in a $2: 1$ mixture $(2 \mathrm{~mL})$ of $\mathrm{MeOH}$ and $\mathrm{CH}_{2} \mathrm{Cl}_{2}$ and cooled to 78 " $\mathrm{C}$ in a dry ice-acetone bath. Ozone was bubbled through the solution until a blue color persisted. Nitrogen was then bubbled through the solution until it 
turned to colorless and $\mathrm{NaBH}_{4}(5 \mathrm{mg})$ was added. The reaction mixture was allowed to wann slowly to room temperature. After $\mathbf{l} \mathrm{h}$. the solution was diluted with AcOEt (2 mL) and quenched with saturated $\mathrm{NaHCO}_{3}$ solution (l mL). The organic portion was decanted and the aqueous portion was washed with $\mathrm{Et}_{2} \mathrm{O}(3 \times 3 \mathrm{~mL})$. The combined organic portions were then washed with brine. The residue was dried ( $\left.\mathrm{MgSO}_{1}\right)$ and concentrated under reduced pressure to yield a colorless residue ( $13 \mathrm{mg}$ ). This residue dissolved in $3 \mathrm{~mL}$ of THF was treated with $\mathrm{LiAlH}_{4}(5 \mathrm{mg})$. After stirring for $10 \mathrm{~h}$, the reaction was quenched with $\mathrm{Na}_{2} \mathrm{SO}_{4}$. $10 \mathrm{H}_{2} \mathrm{O}$. The reaction mixture was filtered through a small column of $\mathrm{Na}_{\beth} \mathrm{SO}_{1}$. and concentrated under reduced pressure to give a diol as colorless oil ( $10 \mathrm{mg}$ ).

A small amount $(6 \mathrm{mg})$ of the diol was dissolved in $\mathrm{CH}_{2} \mathrm{Cl}_{\Sigma}(2 \mathrm{~mL})$ and treated with DMAP (5 mg) and $(R)-$ MTPA-Cl (0.1 mL). After stirring overnight, the reaction was quenched with saturated $\mathrm{NaHCO}_{3}$ solution and extracted with $\mathrm{CH}_{2} \mathrm{Cl}_{2}$. The organic layer was washed with $\mathrm{H}_{2} \mathrm{O}$ and brine. The residue was dried $\left(\mathrm{Na}_{-} \mathrm{SO}_{1}\right)$ and concentrated under reduced pressure. Purification by reversed-phase HPLC (92\% aqueous MeOH) gave an $(R)$-MTPA ester 9a (4.4 mg). 9a: FABMS (pos) $m z 559(\mathrm{M}+\mathrm{Na})$ : 'H NMR $\left(\mathrm{CDCl}_{3}, 400 \mathrm{MHz}\right) \delta 7.683(4 \mathrm{H}, \mathrm{d}, J=7.7 \mathrm{~Hz}, \mathrm{MTPA}-$ phenyl). 7.12-7.05 (6H. m. MTPA-phenyl) +.265 (lH. m. H3). $3.940(2 \mathrm{H}, \mathrm{d}, J=6.1 \mathrm{~Hz} . \mathrm{H}-\mathrm{l}), 3.584$ (3H. MTPA$\left.\mathrm{OCH}_{3}\right), 3.529\left(3 \mathrm{H}, \mathrm{MTPA}-\mathrm{OCH}_{3}\right) .1 .785$ (lH. m, H-2). 1.067 (3H. d. $\left.J=6.8 \mathrm{~Hz}, 2-\mathrm{CH}_{3}\right) .0 .928(3 \mathrm{H}, \mathrm{d} . J=6.9 \mathrm{~Hz}$. $\mathrm{H}-4)$.

On the other hand. the $(S)$-MTPA ester was prepared from the diol ( $+\mathrm{mg}$ ) by the same procedure to prepare the $(R)$ MTPA ester 9a described above. The reaction mixture was purified by reversed-phase HPLC (92\% aqueous $\mathrm{MeOH})$ to give an (S)-MTPA ester 9b (2.1 mg). 9b: FABMS (pos) $m z$ $559(\mathrm{M}+\mathrm{Na})$ ': 'H NMR (CDCl $3.400 \mathrm{MHz}) \delta 7.683(+\mathrm{H} . \mathrm{d} . J$ $=7.7 \mathrm{~Hz}$, MTPA-pheny 1$), 7.12-7.05(6 \mathrm{H}, \mathrm{m}$. MTPA-phenyl). 4.269 (lH. Im, H-3). 3.972 (2H, d. $J=6.1 \mathrm{~Hz}, \mathrm{H}-\mathrm{l}$ ). $3.58+$ $\left(3 \mathrm{H} . \mathrm{MTPA}-\mathrm{OCH}_{3}\right) .3 .529\left(3 \mathrm{H} . \mathrm{MTPA}-\mathrm{OCH}_{3}\right), 1.763(\mathrm{lH}$, Im. H-2). $1.089\left(3 \mathrm{H}, \mathrm{d}, J=6.8 \mathrm{~Hz} .2-\mathrm{CH}_{3}\right) .0 .96+(3 \mathrm{H} . \mathrm{d}, J=$ $6.9 \mathrm{~Hz} . \mathrm{H}-4)$.

Acetonide 10. To a solution of the combined MTPA esters (9a and 9b. $5.1 \mathrm{mg}$ ) in $+: 1$ mixture of $\mathrm{MeOH}$ and $\mathrm{H}_{2} \mathrm{O}(3$ $\mathrm{mL}$ ) was added $\mathrm{LiOH}(5 \mathrm{mg})$. The mixture was stirred at room temperature for 1 day and concentrated under reduced pressure. The residue was diluted with saturated $\mathrm{NaCl}$ solution ( $2 \mathrm{~mL}$ ). The aqueous mixture was acidified to $\mathrm{pH}+$ with $\mathrm{l} \mathrm{N} \mathrm{HCl}$ and extracted with AcOEt $(3 \times 2 \mathrm{~mL})$. The combined organic layers were washed. dried. and concentrated in vactio. The oily residue was purified by column chromatography on silica gel ( $12 \%$ AcOEt/hexane) to afford colorless oil (3.6 mg). The resulting oil was treated with CSA ( $4 \mathrm{mg}$ ) and a $3: 1$ mixture (2 $\mathrm{mL}$ ) of acetone and DMP. After stirring for 2 h. the reaction was quenched with $\mathrm{Et}_{3} \mathrm{~N}$ and concentrated under reduced pressure. The reaction mixture was purified by reversed-phase HPLC (94\% aqueous $\mathrm{MeOH}$ ) to afford acetonide $10(1.8 \mathrm{mg})$. 10: FABMS (pos) $m z \mathrm{l} 67(\mathrm{M}+\mathrm{Na}):{ }^{\prime} \mathrm{H}$ NMR (CDCl $\left.4 .+00 \mathrm{MHz}\right) \delta 3.66$
(lH. dd, $f=13.0,6.6 \mathrm{~Hz} . \mathrm{H}-1) .3 .48$ (lH. dd. $f=13.0 .12 .5$ Hz. H-l). 3.38 (lH, dd. $f=10.3 .7 .3 \mathrm{~Hz} . \mathrm{H}-3$ ). $1.6+(\mathrm{H}, \mathrm{m}$. $\mathrm{H}-2), 1.41$ (3H. s. acetonide $\left.\mathrm{CH}_{3}\right) .1 .36\left(3 \mathrm{H}, \mathrm{s}\right.$. acetonide $\left.\mathrm{CH}_{3}\right)$, $1.13(3 \mathrm{H}, \mathrm{d}, J=7.3 \mathrm{~Hz}, \mathrm{H}-4), 1.02\left(3 \mathrm{H} . \mathrm{d} . J=6.2 \mathrm{~Hz} .2-\mathrm{CH}_{3}\right)$.

p-Bromodibenzoate 11. A suspension of $6(10 \mathrm{mg}) \mathrm{in}$ methanol was treated with hydrogen $(50$ psi) in the presence of $10 \%$ palladium on carbon. After $1 \mathrm{~h}$, the catalyst was filtered and thoroughly washed with methanol. The solution was concentrated completely under reduced pressure. The resulting residue ( $14 \mathrm{mg})$ was continuously treated with acetic anhydride $(2 \mathrm{~mL})$ in $2 \mathrm{~mL}$ of py ridine at room temperature overnight. The reaction misture was cooled to $0^{\circ} \mathrm{C}$ and methanol $(2 \mathrm{~mL}$ ) was added dropwise for $30 \mathrm{~min}$. After exaporation. the mixture was treated with $80 \%$ aqueous $\mathrm{AcOH}(\mathrm{l} \mathrm{mL})$ at $60^{\circ} \mathrm{C}$ for $+\mathrm{h}$. After stirring at room temperature for $\mathrm{l} h$, the reaction mixture was partitioned between $\mathrm{Et}_{2} \mathrm{O}$ and saturated $\mathrm{NaHCO}_{3}$. The organic layer was washed with $\mathrm{H}_{2} \mathrm{O}$ and brine, and dried $\left(\mathrm{Na}_{2} \mathrm{SO}_{1}\right)$. The residue was concentrated under reduced pressure to afford a white amorphous powder. To a solution of this powder ( 17 $\mathrm{mg}$ ) and DMAP (5 $\mathrm{mg}$ ) in $2 \mathrm{~mL}$ of dry pyridine was added p-bromobenzoyl chloride $(5 \mathrm{mg}$ ). The solution was stirred at 40 " $\mathrm{C}$ for 2 days, poured into ice water, and extracted with EtOAc. The organic layer was washed with $5 \% \mathrm{HCl}$. $10 \%$ $\mathrm{NaHCO}_{3}$ and brine, dried $\left(\mathrm{Na}_{2} \mathrm{SO}_{1}\right)$. and concentrated in vacuo. The residue was purified by a series of preparative TLC (10\% AcOEt/hexane) and reversed-phase HPLC (96\% aqueous $\mathrm{MeOH}$ ) to give a major $\mathrm{di}-p$-bromobenzoate (11. $2.6 \mathrm{mg}$ ) as an amorphous solid. 11: FABMS (pos) $m: 799$ $(\mathrm{M}+\mathrm{H})^{\prime}:$ 'H NMR $\left(\mathrm{CDCl}_{3 .} 400 \mathrm{MHz}\right) \delta 7.93(2 \mathrm{H}, \mathrm{d}, j=8.7$ Hz. 4-bromobenzoate), 7.86 (2H. d. $J=8.7 \mathrm{~Hz}, 5$-bromobelzoate), $7.70(2 \mathrm{H}, \mathrm{d}, J=8.7 \mathrm{~Hz}$ 4-bromobenzoate). 7.66 (2H. d. $J=8.7 \mathrm{~Hz}, 5$-bromobenzoate), 4.42 (1H, m. H-5). 4.27 (lH. m. H-t). 3.68 (lH. m. H-ll), 3.54 (lH, m. H-7), $3.16\left(3 \mathrm{H}\right.$. s. $\left.13-\mathrm{OCH}_{3}\right), 3.02\left(3 \mathrm{H}, \mathrm{s}, 1-\mathrm{OCH}_{3}\right), 2.40(\mathrm{lH} . \mathrm{dd}$. $J=13.6,8.5 \mathrm{~Hz}, \mathrm{H}-\mathrm{l} 0), 2.29(\mathrm{lH}, \mathrm{dd}, J=13.5,4.1 \mathrm{~Hz}, \mathrm{H}-$ 12). 2.20 ( $\mathrm{HH}$. dd. $J=14.2 .3 .3 \mathrm{~Hz}, \mathrm{H}-8$ ). 2.14 (3H. s. $7-$ OAc). 2.10 (lH. dd. $J=13.6 .3 .0 \mathrm{~Hz} . \mathrm{H}-10) .2 .06(3 \mathrm{H}$. s. $11-$ OAc). 1.99 (2H. t. $J=7.2 \mathrm{~Hz} . \mathrm{H}-2$ ). 1.92 ( $\mathrm{lH}$. dd. $J=13.5$. $6.5 \mathrm{~Hz}, \mathrm{H}-12), 1.80$ ( $1 \mathrm{H}, \mathrm{dd}, J=1+2.8 .0 \mathrm{~Hz} . \mathrm{H}-8) .1 .6 \mathrm{l}$ (2H. m, H-3). 1.38 ( $\mathrm{HH}$. ddd. $J=1+.8,11.6 .9 .9 \mathrm{~Hz}, \mathrm{H}-6$ ). 1.20 ( $1 \mathrm{H}$, ddd. $J=14.8 .2 .9 .2 .0 \mathrm{~Hz} . \mathrm{H}-6$ ).

Tetraacetyl derivative 12. Tetrin B (1.20 mg) dissolved in $2 \mathrm{~mL}$ of $\mathrm{MeOH}$ was treated with diazomethane at room temperature for $l \mathrm{~h}$. The solution was exaporated under reduced pressure to give a reaction mixture. This mixture was then diluted with ethyl acetate $(2 \mathrm{~mL})$ and washed with $\mathrm{H}_{2} \mathrm{O}(2 \mathrm{~mL})$ and brine $(2 \mathrm{~mL})$. A yellow solid residue (14 my) was obtained by silica gel columu chromatography ( $15 \%$ AcOEt/hexane). The yellow residue in $2 \mathrm{~mL}$ of pyridine was continuously treated with acetic anhydride $(2 \mathrm{~mL})$ at room temperature overnight. After exaparation the reaction residue was dissolved in $5 \% \mathrm{HCl}-\mathrm{MeOH}(1 \mathrm{~mL})$ and heated under reflux for $+\mathrm{h}$. The reaction mixture was adjusted to neutral with $\mathrm{AgNO}_{3}$ and then filtered. The filtrate was evaporated under reduced pressure and the residue was adsorbed on a silica column ( $15 \%$ AcOEt/hexane) to yield 
deglycosylated tetracyl tetrin B 12 (9 $\mathrm{mg}$ ). 12: FABMS (pos) $m z 757(\mathrm{M}+\mathrm{Na})^{\prime} .735(\mathrm{M}+\mathrm{H}){ }^{\circ}:{ }^{l} \mathrm{H} \mathrm{NMR}\left(\mathrm{CDCl}_{5}, 400\right.$ $\mathrm{MHz}) \delta 6.87(\mathrm{lH}$. dd. $J=15.5 .3 .2 \mathrm{~Hz}, \mathrm{H}-3) .6 .46-6.05(6 \mathrm{H}$, complex). $5.9+$ (lH, dd. $J=15.6,8.9 \mathrm{~Hz}, \mathrm{H}-\mathrm{l6}) .5 .82(\mathrm{lH}, \mathrm{d}$, $J=15.5 \mathrm{~Hz}, \mathrm{H}-2), 5.70(1 \mathrm{H}, \mathrm{dd} . J=15.0,6.8 \mathrm{~Hz} . \mathrm{H}-23)$. 4.86 (1H. ddd. $J=10.8,10.2,4.8 \mathrm{~Hz}, \mathrm{H}-1 \mathrm{l}) .+72(\mathrm{lH} . \mathrm{m}$. H25). 4.60 (1H. dd, $J=11.2,3.2 \mathrm{~Hz}, \mathrm{H}-4), 4.49$ (1H. ddd. $y=$ 11.2. 9.6. $2.0 \mathrm{~Hz} . \mathrm{H}-5),+.37$ (1H. m, H-7), +.26 (1H. ddd, $J$ $=10.2,8.5,1.5 \mathrm{~Hz}, \mathrm{H}-13) .3 .54$ (1H, ddd, $J=8.9,4.0,2.5$ Hz. H-15), 3.46 (3H. s. 12-CO-CH3). 2.39 (lH. m. H-24), $2.28(1 \mathrm{H}, \mathrm{t} . J=10.2 \mathrm{~Hz} . \mathrm{H}-12$ ). 2.20 (lH. ddd. $J=15.3 .8 .5$, $4.0 \mathrm{~Hz}, \mathrm{H}-\mathrm{l} 4), 2.14(3 \mathrm{H}, \mathrm{s} .5-\mathrm{OAc}) .2 .11$ (3H, s, 7-OAc), $2.09(3 \mathrm{H}, \mathrm{s} .1 \mathrm{l}-\mathrm{OAc}) \cdot 2.07(3 \mathrm{H}, \mathrm{s}, 4-\mathrm{OAc}), 1.91(1 \mathrm{H}, \mathrm{dd}, J=$ 12.6. $4.8 \mathrm{~Hz} . \mathrm{H}-10$ ). 1.80 (1H. dd. $J=13.6 .2 .1 \mathrm{~Hz} . \mathrm{H}-8$ ), 1.74 (1H. dd. $J=12.6,10.8 \mathrm{~Hz} . \mathrm{H}-10), 1.64(1 \mathrm{H}, \mathrm{dd} . J=$ 13.6. $11.0 \mathrm{~Hz}, \mathrm{H}-8$ ), 1.55 ( $\mathrm{lH}$. ddd, $J=15.3,2.5 .1 .5 \mathrm{~Hz} . \mathrm{H}-$ 14). 1.47 (1H, m. H-6), 1.26 (1H, m. H-6), 1.12 (3H. d. $J=$ $6 .+\mathrm{Hz} . \mathrm{H}-26) .1 .04$ (3H. d. $J=6.1 \mathrm{~Hz} .2+-\mathrm{CH}_{3}$ ).

MTPA esters 13a and 13b. To a stirred solution of compound $12(+\mathrm{mg})$ in $0.5 \mathrm{~mL}$ of $\mathrm{CH}_{2} \mathrm{Cl}_{2}$ were added DMAP $(6 \mathrm{mg})$ and $(R)$-MTPA-Cl $(0.1 \mathrm{~mL})$. After $18 \mathrm{~h}$ the reaction mixture was concentrated under reduced pressure. The residue was dissolved in etlyy acetate and the organic solution was washed successively with water. dried over $\mathrm{Na}_{2} \mathrm{SO}_{4}$. filtered. and concentrated under reduced pressure. The residue was subjected to reversed-phase HPLC $(95 \%$ $\mathrm{MeOH})$ to give $(R)$-MTPA ester 13a (2.6 mg). 13a: FABMS (pos) $m z^{95}(\mathrm{M}+\mathrm{H})^{\prime}:{ }^{1} \mathrm{H} \mathrm{NMR}\left(\mathrm{CDCl}_{3,} 400 \mathrm{MHz}\right) \delta 7.326$ (5H. complex, MTPA-phenyl). 6.783 (H. dd. $J=15.5 .3 .2$ Hz. H-3). 6.540-6.220 (6H, complex). 6.083 (1H. dd. $j=$ 15.6. $8.9 \mathrm{~Hz}, \mathrm{H}-16$ ). $5.86+$ (lH. d. $J=15.5 \mathrm{~Hz}, \mathrm{H}-2$ ). 5.686 (lH. dd. $J=15.0,6.8 \mathrm{~Hz} . \mathrm{H}-23$ ). $4.7+3$ (lH. ddd. $J=10.8$. 10.2. $4.8 \mathrm{~Hz} . \mathrm{H}-1 \mathrm{l}) .4 .549$ (1H. m. H-25). 4.638 (lH. dd. $J=$ 11.2. $3.2 \mathrm{~Hz} . \mathrm{H}-4) .+.552\left(3 \mathrm{H}, \mathrm{MTPA}-\mathrm{OCH}_{3}\right)+4.482(\mathrm{lH}$, ddd. $J=11.2 .9 .6 .2 .0 \mathrm{~Hz} . \mathrm{H}-5) .+.38 \mathrm{l}(\mathrm{lH} . \mathrm{m}, \mathrm{H}-7) .+254$ (1H. dd $J=10.2 .1 .6 \mathrm{~Hz}, \mathrm{H}-13$ ). 4.146 (1H. ddd, $J=8.9$, $40.2 .5 \mathrm{~Hz}, \mathrm{H}-15) .3 .443\left(3 \mathrm{H}, \mathrm{s} .12-\mathrm{CO}_{2} \mathrm{CH}_{3}\right) .2 .386(\mathrm{lH}, \mathrm{m}$. $\mathrm{H}-24) .2 .168$ (1H, t $J=10.2 \mathrm{~Hz}, \mathrm{H}-12$ ). 2.252 ( $1 \mathrm{H}$, ddd. $J=$ 15.3. 8.5.4.0 Hz. H-l+). $2.10 \mathrm{l}$ (3H, s. 5-OAc). 2.087 (3H, s, 7-OAc). $2.08+$ (3H. s. 11-OAc). 2.062 (3H. s. 4-OAc). 1.94t (lH. dd. $J=12.6 .4 .8 \mathrm{~Hz} . \mathrm{H}-10$ ), 1.862 (1H. dd. $J=13.6 .2 .1$ Hz. H-8) 1.756 (lH. dd. $J=12.6 .10 .8 \mathrm{~Hz}, \mathrm{H}-10$ ), 1.634 (lH. dd. $J=13.6,11.0 \mathrm{~Hz} . \mathrm{H}-8$ ). $1.50 \mathrm{l}$ (1H. ddd $J=15.3$. $2.5,1.6 \mathrm{~Hz} . \mathrm{H}-14), 1.398$ (1H, m. H-6), 1.247 (lH. m. H-6), 1. $166(3 \mathrm{H}$, d. $J=6.4 \mathrm{~Hz}, \mathrm{H}-26) .1 .062(3 \mathrm{H} . \mathrm{d} . J=6.1 \mathrm{~Hz}$. $\left.24-\mathrm{CH}_{3}\right)$.

The $(S)$-MTPA ester was prepared from acetonide $12(4$ $\mathrm{mg}$ ) by the same procedure used to prepare the $(R)$-MTPA ester 13a described above. The reaction mixture was purified by reversed-phase HPLC $(95 \% \mathrm{MeOH})$ to afford an $(S)$ MTPA ester $13 \mathrm{~b}$ (2.2 mg). 13b: FABMS (pos) $m=95 \mathrm{l}(\mathrm{M}+$ $\mathrm{H})$ : 'H NMR (CDCl, $400 \mathrm{MHz}) \delta 7.326(5 \mathrm{H}$, complex, MTPA-phenyl), 6.783 (lH. dd $J=15.5 \mathrm{~Hz} .3 .2 . \mathrm{H}-3$ ).
$6.560-6.210(6 \mathrm{H}$, complex). $6.051(\mathrm{lH} . \mathrm{dd} . j=15.6 .8 .9 \mathrm{~Hz}$, $\mathrm{H}-16), 5.86 \mathrm{l}(\mathrm{lH}, \mathrm{d}, f=15.5 \mathrm{~Hz}, \mathrm{H}-2), 5.682$ (1H. dd, $f=$ $15.0 .6 .8 \mathrm{~Hz} . \mathrm{H}-23) .4 .751$ (1H. ddd. $f=10.8$. $10.2 \mathrm{~Hz} .4 .8$. $\mathrm{H}-\mathrm{ll}), 4.5+7(\mathrm{lH}, \mathrm{m}, \mathrm{H}-2.5), 4.639(\mathrm{lH}, \mathrm{dd} . J=11.2 .3 .2 \mathrm{~Hz}$. $\mathrm{H}-4)+.552\left(3 \mathrm{H}, \mathrm{MTPA}-\mathrm{OCH}_{3}\right), 4.483(\mathrm{lH}$. ddd, $J=11.2$. 9.6. $2.0 \mathrm{~Hz} . \mathrm{H}-5$ ). 4.379 (lH. m. H-7). 4.272 (1H. dd. $f=$ 10.2. $1.6 \mathrm{~Hz} . \mathrm{H}-13$ ). 4.149 (1H. ddd. $f=8.9 .4 .0 .2 .5 \mathrm{~Hz}$. H15). $3.460\left(3 \mathrm{H}, \mathrm{s}, 12-\mathrm{CO}_{2} \mathrm{CH}_{3}\right) .2 .38+(\mathrm{lH}, \mathrm{m}, \mathrm{H}-24), 2.191$ (lH. $\mathrm{t} . J=10.2 \mathrm{~Hz}, \mathrm{H}-12$ ), 2.274 (1H. ddd, $J=15.3,8.5,4.0$ Hz. H-l4), 2.103 (3H. s. 5-OAc), 2.086 (3H. s. 7-OAc), 2.099 (3H. s. 1l-OAc). 2.060 (3H.s. 4-OAc). 1.954 (1H. dd. $J=12.6,4.8 \mathrm{~Hz} . \mathrm{H}-10), 1.869$ (1H. dd, $J=13.6,2.1 \mathrm{~Hz}, \mathrm{H}-$ 8). $1.768(1 \mathrm{H}, \mathrm{dd}, J=12.6,10.8 \mathrm{~Hz}, \mathrm{H}-10), 1.6+1$ (1H. dd. $f$ $=13.6 .11 .0 \mathrm{~Hz}, \mathrm{H}-8) .1 .519(1 \mathrm{H}$. ddd. $f=15.3 .2 .5,1.6 \mathrm{~Hz}$. $\mathrm{H}-14), 1.400$ (lH. m. H-6), 1.248 (1H, m. H-6), $1.163(3 \mathrm{H}$, d. $J=6.4 \mathrm{~Hz} . \mathrm{H}-26), 1.060\left(3 \mathrm{H} . \mathrm{d}, J=6.1 \mathrm{~Hz}, 24-\mathrm{CH}_{3}\right)$.

Acknowledgment. This work was supported by the Korea Science and Engineering Foundation (KOSEF) through the Advanced Material Research Center for Better Environment. Hanbat National University. We thank Dr. U.C. Lee of Korea Ginseng \& Tobacco Research lnstitute for NMR and mass measurements.

\section{References}

1. Rinchart, K. I... Ir: Germatn. V. F.: Tucker, W. P.: Gottlicb, D. Jushus Liebigs fm. Chem. 1963.668. 77.

2. Rineharl. K. L.. Ir.: lucker. W. I': I'andey. R. C.J. Am (hem. Soc. 1971.93.3747

3. P'andey. R. C.: Rinehart. K. L.. Jr. J. Inthohtics 1977. 30.158.

4. Patrick. J. B.: Williams, R. R.: Wolt, C. F.: Weeb. J. S. J. .tm. Chem. Soc 1958. 80, 6688.

5. (a) Domberger, K.: Thrum, II.: Radies, I. Fttrohedron 1979, 35 , 1851. (b) Ceder. O.: Hansson. B.: Rapp. U. Tetrahtedmon 1977. 33. 2703.

6. Oppong. L.: Pauls. H. W.: Fraser-Reid. B. J. Chem. Soc. ('he'm. Commar. 1986. 1241

7. Duplantic, A. J.: Masamunc. S. J. Am. Chem. Soc 1990. 112. 7079 .

8. (a) Lancelin. I. M.: Beau. J. M. J. finer (hem Soc 1990. //2. 4060. (b) Lancelin. J. M.: Beau. I. M. Bull. Soc. (him. Fr. 1995. 132.215 .

9. Riu. G.: Choi. W.C.: I Iwang, S.: Yeo, W.-I I.: I.ec. C.-S.: Kim. S.-K. J. Kat. Prod 1999. 62. 917

10. 10. (a) Ryclunorsky, S. D.: Skalitzky, D. I. Fetrohedron Let. 1990. 31. 945. (b) Rychnorsky. S. D.: Rogers. B.: Yang. G. f. Org. Chem. 1993. 58. 3511 . (c) Evans. D. A.: Rieger. D. L.: Gage. I. R. Tetrahedron lett 1990. 31.7099.

11. Chuvile, I.: Dana, G.: Monot, M. R. Bull Soc. Chm Soc. 1976. 9. 3.300

12. (a) Dalc, I. A.: Mosher, II. S. J. Org. Chem 1973, 95, 512. (b) Ohtani. I.: Kusumi. T.: Kahman. Y.: Kakisawa. H. J. (m. Chem. Soc. 1991. 73. 4092. (c) Kusumi. T.: Fukushima. T:: Ohtami. I.: Kakisawa. H. Tetwhedron I.eft 1991. 32. 2939.

1.3. Donberger. K.: Thrum, H.: Radics, I. Tetrahedrom 1979, 35. 1851 .

14. Radics, I.: Ineze, M.: Domberger, K.: Thrum, HI. Tefrahedon 1982. $3 \% .183$. 